\title{
A Constrained Conjugate Gradient Method for Solving the Magnetic Field Boundary Integral Equation
}

\author{
Peter M. van den Berg, Erdal Korkmaz, and Aria Abubakar, Member, IEEE
}

\begin{abstract}
It is well-known that electromagnetic solutions of boundary integral equations for perfectly electrically conducting scatterers are nonunique for those frequencies which correspond to interior resonances of the scatterer. In this paper a simple and efficient computational method is developed, in which the interior integral representations, required to hold on an interior closed surface, are used as a sufficient constraint to restore uniqueness. We use the interior equations together with the second kind magnetic field integral equation, so that the ill-posedness of the interior equations does not give a problem. We develop a constrained conjugate gradient method that minimizes a cost functional consisting of two terms. The first term is the error norm with respect to the magnetic field boundary integral equation, while the second term is the error norm with respect to the interior equations over a closed interior surface, which is chosen as small as possible. Some numerical examples show the robustness and efficiency of the pertaining computational procedure.
\end{abstract}

Index Terms-Boundary integral equation, conjugate gradient, interior resonance, uniqueness.

\section{INTRODUCTION}

$\mathbf{T}$ HE scattering of an electromagnetic wave by a perfectly conducting closed body is usually formulated by the electric field boundary integral equations (EFIE) and the magnetic field boundary integral equation (MFIE), see [1], [2]. A disadvantage of the use of the boundary type of integral equations is related to the uniqueness of their solution. Although the theoretical problem of the nonuniqueness has been well understood [3], [4], the problem is often not anticipated in advance. Development involving the numerical solution of integral equations typically concentrates on the treatment of small or moderately-sized scatterers, at least for the initial testing and validation of the formulations. Then, the solution is obtained by direct matrix inversion of the discretized equations and the electric field and magnetic field integral equations produce perfect solutions as long as the frequency of operation does not coincide with an eigenfrequency of an interior resonance [5]. For

Manuscript received February 20, 2002; revised April 17, 2002. This work was supported by the Toegepast-Natuurwetenschappelijk Onderzoek (TNO) Physics and Electronics Laboratory, The Hague, The Netherlands.

P. M. van den Berg is with the Laboratory of Electromagnetic Research, Delft University of Technology, 2628 CD Delft, The Netherlands (e-mail: p.m.vandenberg@its.tudelft.nl).

E. Korkmaz is with the TNO Physics and Electronics Laboratory, Oude Waalsdorperweg 63, 2509 JG, The Hague, The Netherlands.

A. Abubakar is with Schlumberger-Doll Research, Ridgefield, CT 06877

USA (e-mail: aabubakar@ ridgefield.oilfield.slb.com).

Digital Object Identifier 10.1109/TAP.2003.812275 electrically large objects, the number of unknowns becomes so large that direct matrix inversion is not feasible, and we adopt the conjugate gradient method [6] as an iterative solver of the discretized integral equation. But within some frequency band around the eigenfrequency of an internal resonance the convergence rate of this iterative scheme decreases dramatically and the numerical results are not reliable.

Mitzner [7] recommended the use of a linear combination of the EFIE and the MFIE as a remedy to overcome nonuniqueness problems at eigenfrequencies of interior resonances. The resulting formulation is known as the combined-field integral equation [8], [9]. But the drawback is the significant increase of computation time for the calculation of the matrix elements of the two sets of discretized equations. Yaghjian [10] augments the MFIE with the normal projection of the integral equation, while recently a generalized minimal residual (GMRES) iterative solution of these augmented system is discussed by Makarov and Vedantham [11]; the latter authors remark that their approach is not expected to completely eliminate the numerical problems due to the spurious resonances. Another approach is the use of dual surfaces [12], [13] to create a well-conditioned problem. The dual surface is located at approximately one quarter wavelength inside the actual scatterer surface. The disadvantage is the extra computation of the matrix elements with respect to this interior dual surface. The objective of the present paper is to minimize these extra computations by taking a smaller interior surface, without losing uniqueness. This is achieved by using the MFIE in combination with interior integral representations (often called null-field equations) obtained by applying Oseen's extinction theorem [14].

In contrast to the dual surface equation used in [12] and [13], where the tangential components of the EFIE are used, we use the three components of the null-field equations of the magnetic type. This guarantees uniqueness, irrespectively of the choice of the interior closed surface. This idea is inspired by the so-called CHIEF method in acoustics, originated by Schenck [15], and the work of Mittra and Klein [16] in electromagnetics, using an over specification with these null-field equations throughout the interior region. Although we are dealing with a unique solution when all interior points are taking into account, for computational reasons the number of interior points (and, hence, extra equations) should be limited. The preselection of the interior points must then be based on some a priori information about the occurrence and nature of the interior resonance. This problem is avoided by assuming that these interior points span a 


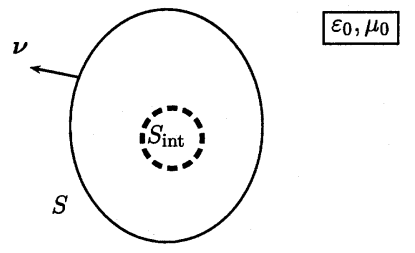

Fig. 1. Scattering configuration with boundary surface $S$ and an interior surface $S_{\text {int }}$.

surface that encloses a nonvanishing subdomain. Observing that on this closed interior surface a convergent expansion of the incident field and the Green function in spherical regular vector eigenfunctions [17] exists, we may conclude that the interior field representations hold everywhere in the interior of the object and hence uniqueness is guaranteed. We therefore replace the preselection of interior points by this null-field integral equation over a closed interior surface. We first use this null-field integral equation, to be imposed on a closed interior surface, as a criterion to monitor the error in the solution. Subsequently we use it as a sufficient constraint to the MFIE to guarantee a unique solution. Combining this first kind null-field integral equation together with the second kind MFIE and normalizing both equations properly, the ill-posedness of the first kind integral equation over the interior surface is not important and we may choose the interior surface as small as possible, as long as the field variation over this surface remains computable within the degree of accuracy.

\section{MAGNETIC Field EQuATIONS}

Consider the electromagnetic scattering by an arbitrary perfectly conducting object with a closed boundary surface $S$ (Fig. 1). The spatial position is denoted by $\boldsymbol{x}$. The total electromagnetic field $\{\boldsymbol{E}, \boldsymbol{H}\}$ can be written as the sum of an incident field $\left\{\boldsymbol{E}^{i}, \boldsymbol{H}^{i}\right\}$ and a scattered field $\left\{\boldsymbol{E}^{s}, \boldsymbol{H}^{s}\right\}$. All fields have a time factor $\exp (-i \omega t)$.

In view of its simplicity we take the MFIE as point of departure, in our notation written as

$$
\begin{aligned}
& \boldsymbol{\nu}_{p} \times \boldsymbol{H}^{i}\left(\boldsymbol{x}_{p}\right)=\frac{1}{2} \boldsymbol{\nu}_{p} \times \boldsymbol{H}\left(\boldsymbol{x}_{p}\right) \\
&-\boldsymbol{\nu}_{p} \times\left[\boldsymbol{\nabla}_{p} \times \int_{S} G\left(\boldsymbol{x}-\boldsymbol{x}_{p}\right) \boldsymbol{\nu} \times \boldsymbol{H}(\boldsymbol{x}) d A\right]
\end{aligned}
$$

for $\boldsymbol{x}_{p} \in S$, while $\boldsymbol{\nu}$ and $\boldsymbol{\nu}_{p}$ are unit vectors along the normal to $S$ at position $\boldsymbol{x}$ and $\boldsymbol{x}_{p}$. The gradient $\nabla_{p}$ represents the spatial differentiation with respect $\boldsymbol{x}_{p}$. The Green function is given by $G\left(\boldsymbol{x}-\boldsymbol{x}_{p}\right)=\exp \left(i k_{0}\left|\boldsymbol{x}-\boldsymbol{x}_{p}\right|\right) / 4 \pi\left|\boldsymbol{x}-\boldsymbol{x}_{p}\right|$, where $k_{0}$ is the wave number in the embedding medium.

To overcome the nonuniqueness of the MFIE we require consistency inside the scattering object. For points $\boldsymbol{x}_{q}$ inside the object we arrive at the following interior equations:

$$
\boldsymbol{H}^{i}\left(\boldsymbol{x}_{q}\right)=-\nabla_{q} \times \int_{S} G\left(\boldsymbol{x}-\boldsymbol{x}_{q}\right) \boldsymbol{\nu} \times \boldsymbol{H}(\boldsymbol{x}) d A .
$$

However, it is not necessary to consider all the interior points. Let us consider a closed surface $S_{\text {int }}$ that completely lies in the interior of the scatterer. When (2) is satisfied on this interior surface, both for the incident field and the Green function we can use a convergent expansion in regular vector eigenfunctions in- side and on this closed surface [17], and we may conclude that these equation holds inside $S_{\text {int }}$ as well. Subsequently, in view of analytic continuation, (2) holds everywhere in the interior of the object. Hence, the satisfaction of the interior representation on a surface enclosing any nonvanishing interior subdomain is a sufficient condition for uniqueness of the solution of the surface field $\boldsymbol{\nu} \times \boldsymbol{H}$ on the boundary of the object. In fact, (2) to be satisfied for all points on an interior surface $S_{\text {int }}$ can be considered as a first kind integral equation, with a unique solution [17]. However, we do not advocate to solve this ill-posed equation numerically, but we use this equation in combination with the MFIE of (1).

\section{Conjugate Gradient Solution}

We discretize the boundary surface $S$ into $N$ planar, triangular surface elements $S_{n}$ with area $A_{n}$. The position vectors of the vertices on a patch $S_{n}$ are denoted as $\boldsymbol{x}_{\mathrm{n}, \mathrm{i}},\{i=1,2,3\}$. We use a linear interpolation function to express any quantity defined on $S_{n}$. We are interested in objects which are large compared to the wavelength of the incident field. In practice, we need at least five to six discretization points per wavelength [20]. Hence, for large objects, the storage of the system matrix and its inversion become unrealistic even with the present-day computer power. We therefore aim for an iterative solution of the system of equations. Starting with the (high-frequency) physical-optics approximation [1], where we take the surface field as $\boldsymbol{\nu} \times \boldsymbol{H}=2 \boldsymbol{\nu} \times \boldsymbol{H}^{i}$ on the "illuminated" part of the object surface, and equal to zero on the "dark" part of the object, we anticipate that a conjugate gradient scheme [18] would be able to compute an improved approximation efficiently. To this end, we use an error criterion based on the normalized error norm

$$
E R R_{S}^{2}=\frac{\left\|\boldsymbol{\nu} \times \boldsymbol{H}^{i}-\mathcal{L}[\boldsymbol{\nu} \times \boldsymbol{H}]\right\|_{S}^{2}}{\left\|\boldsymbol{\nu} \times \boldsymbol{H}^{i}\right\|_{S}^{2}}
$$

where the operator $\mathcal{L}$ acting on a tangential vector function $[\boldsymbol{\nu} \times$ $\boldsymbol{H}$ ] on $S$ follows from the MFIE (in discrete form) as:

$$
\begin{aligned}
\mathcal{L}[\boldsymbol{\nu} \times \boldsymbol{H}] & \equiv \frac{1}{2}[\boldsymbol{\nu} \times \boldsymbol{H}]\left(\boldsymbol{x}_{\mathrm{m}, \mathrm{j}}\right) \\
& +\boldsymbol{\nu}_{m} \times \sum_{n=1}^{N} \sum_{i=1}^{3} \frac{A_{n}}{3} \nabla \mathcal{G}\left(\boldsymbol{x}_{\mathrm{n}, \mathrm{i}}-\boldsymbol{x}_{\mathrm{m}, \mathrm{j}}\right) \times[\boldsymbol{\nu} \times \boldsymbol{H}]\left(\boldsymbol{x}_{\mathrm{n}, \mathrm{i}}\right) .
\end{aligned}
$$

The gradient acting on the Green function (in weak form) denotes the spatial differentiation with respect to $\boldsymbol{x}_{\mathrm{n}, \mathrm{i}}$. The norm of on $S$ is defined as

$$
\begin{aligned}
\|\boldsymbol{\nu} \times \boldsymbol{H}\|_{S}^{2} & =\langle[\boldsymbol{\nu} \times \boldsymbol{H}],[\boldsymbol{\nu} \times \boldsymbol{H}]\rangle_{S} \\
& =\sum_{m=1}^{N} \sum_{j=1}^{3} \frac{A_{m}}{3}[\boldsymbol{\nu} \times \boldsymbol{H}]\left(\boldsymbol{x}_{\mathrm{m}, \mathrm{j}}\right) \cdot \overline{[\boldsymbol{\nu} \times \boldsymbol{H}]\left(\boldsymbol{x}_{\mathrm{m}, \mathrm{j}}\right)}
\end{aligned}
$$

where the overline denotes complex conjugate. Further, we need the adjoint operator, which is obtained (in discrete form) as

$$
\begin{aligned}
\mathcal{L}^{*}[\boldsymbol{\nu} \times \boldsymbol{f}] & \equiv \frac{1}{2}[\boldsymbol{\nu} \times \boldsymbol{f}]\left(\boldsymbol{x}_{\mathrm{m}, \mathrm{j}}\right) \\
+\boldsymbol{\nu}_{m} & \times \sum_{n=1}^{N} \sum_{i=1}^{3} \frac{A_{n}}{3} \nabla \overline{\mathcal{G}}\left(\boldsymbol{x}_{\mathrm{n}, \mathrm{i}}-\boldsymbol{x}_{\mathrm{m}, \mathrm{j}}\right) \times[\boldsymbol{\nu} \times \boldsymbol{f}]\left(\boldsymbol{x}_{n, i}\right) .
\end{aligned}
$$




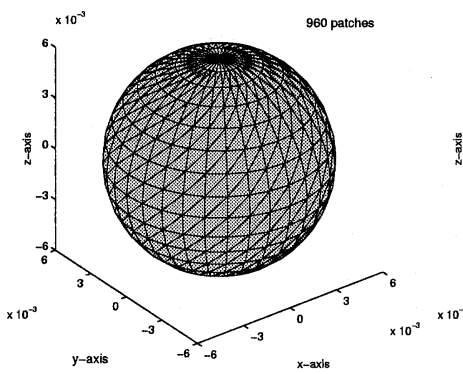

(a)

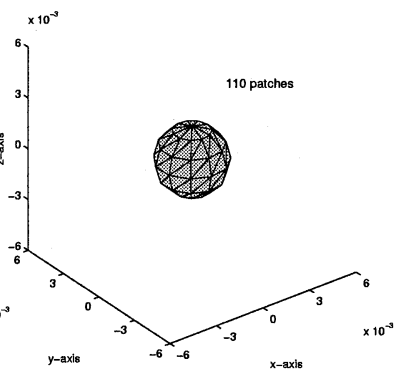

(b)
Fig. 2. (a) Sphere of radius $a$ discretized in 960 patches and (b) interior sphere of radius $a / 3$ discretized in 110 patches.

In fact, the conjugate gradient scheme solves iteratively the symmetrized integral equation

$$
\mathcal{L}^{*} \mathcal{L}[\boldsymbol{\nu} \times \boldsymbol{H}]=\mathcal{L}^{*}\left[\boldsymbol{\nu} \times \boldsymbol{H}^{i}\right], \quad \text { on } S .
$$

If and only if MFIE is uniquely solvable, the symmetrized operator $\mathcal{L}^{*} \mathcal{L}$ does not have a null-space and is positive, so that the conjugate gradient scheme (for the nondiscretized problem) converges to the exact solution [21].

\section{A. Additional Error Criterion}

To monitor the solution of MFIE, we use the interior representation at points on the discretized surface $S_{\text {int }}$. We, therefore, define an additional error criterion

$$
\mathrm{ERR}_{S_{\text {int }}}=\frac{\left\|\boldsymbol{H}^{i}-\mathcal{L}_{\text {int }}[\boldsymbol{\nu} \times \boldsymbol{H}]\right\|_{S_{\text {int }}}}{\left\|\boldsymbol{H}^{i}\right\|_{S_{\text {int }}}}
$$

where the norm over the interior surface $S_{\text {int }}$ is defined as

$$
\left\|\boldsymbol{H}^{i}\right\|_{S_{\mathrm{int}}}^{2}=\left\langle\boldsymbol{H}^{i}, \boldsymbol{H}^{i}\right\rangle=\sum_{m=1}^{N_{\mathrm{int}}} A_{m} \boldsymbol{H}^{i}\left(\boldsymbol{b}_{m}\right) \cdot \overline{\boldsymbol{H}^{i}\left(\boldsymbol{b}_{m}\right)},
$$

where the interior surface $S_{\text {int }}$ is subdivided into $N_{\text {int }}$ planar triangles, with area $A_{m}$ and barycenter $\boldsymbol{b}_{m}=(1 / 3) \sum_{j=1}^{3} \boldsymbol{x}_{\mathrm{m}, \mathrm{j}}$. The definition of $\mathcal{L}_{\text {int }}$ immediately follows from the right-hand side of (2) as

$$
\mathcal{L}_{\text {int }}[\boldsymbol{\nu} \times \boldsymbol{H}] \equiv \sum_{n=1}^{N} \sum_{i=1}^{3} \frac{A_{n}}{3} \nabla \mathcal{G}\left(\boldsymbol{x}_{\mathrm{n}, \mathrm{i}}-\boldsymbol{b}_{m}\right) \times[\boldsymbol{\nu} \times \boldsymbol{H}]\left(\boldsymbol{x}_{\mathrm{n}, \mathrm{i}}\right) .
$$

This operator $\mathcal{L}_{\text {int }}$ maps $S$ into $S_{\text {int }}$. We shall use this additional error norm as an extra check of the numerical results. In order to guarantee that in the discretized counterpart of $S_{\text {int }}$ the field variation over the interior surface is computable within the present computer accuracy, we anticipate that it is sufficient that $S_{\text {int }}$ encloses a sphere with a diameter of a half wavelength, and on $S_{\text {int }}$ we take a discretization of five to six points per wavelength. Taking a larger interior surface and/or a finer discretization is always allowed, but it will increase the computation time without any benefit.

\section{B. Numerical Example of a Sphere}

As a first test example we consider the scattering by a perfectly conducting sphere with radius $a$. We assume that its center coincides with the origin of our coordinate system. A plane wave propagating in the negative $z$ direction is incident upon the sphere. We take the polarization of the electric field in the

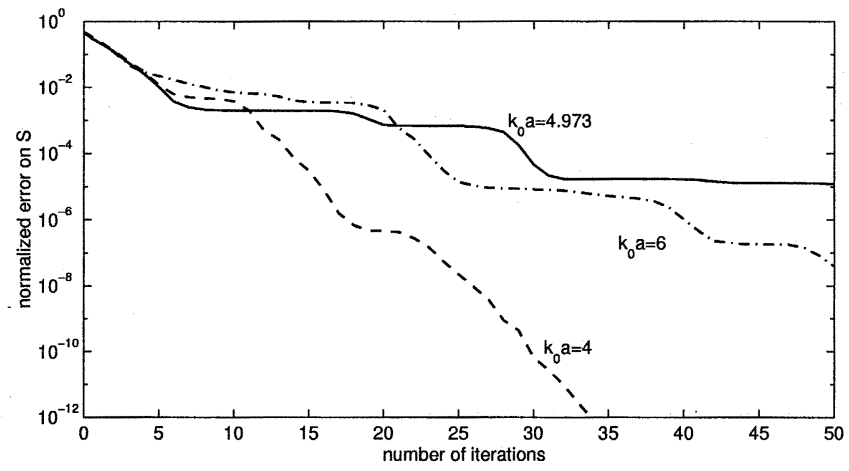

(a)

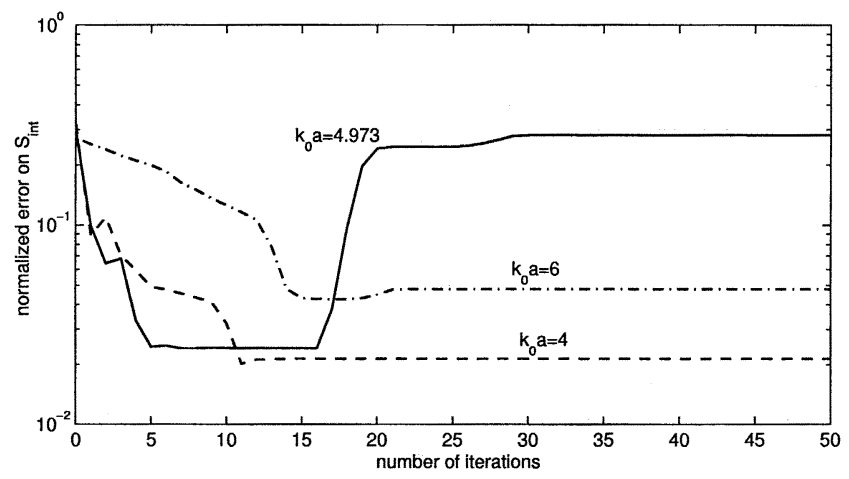

(b)

Fig. 3. (a) Normalized errors on $S$ and (b) $S_{\text {int }}$ in conjugate gradient scheme.

$x$ direction and in the $y$ direction, respectively. In the several figures in this paper, we present the error only for the case that the electric field of the incident wave is polarized in the $y$ direction. We consider three frequencies of operation, such that the normalized wave numbers are $k_{0} a=4,4.973$ and 6, respectively. At $k_{0} a=4.973$ a spurious solution can occur due to the nonuniqueness of the MFIE at the eigenfrequency of an interior resonance. We discretize the boundary surface $S$ of the sphere in 960 plane triangles patches [see Fig. 2(a)].

To investigate the problem of the occurrence of a spurious field solution in more detail, we compute our additional error norm over an interior surface $S_{\text {int }}$ as well. Here, we take a sphere with a radius of $a / 3$, subdivided in 110 plane triangular patches [see Fig. 2(b)]. The extra computation time to compute the error on $S_{\text {int }}$ is less than 3\%. In Fig. 3(a) we present the normalized error norm, $E R R_{S}$, minimized by the conjugate gradient scheme, as a function of the number of iterations. We observe that the error on $S$ in the conjugate gradient scheme remains decreasing, although at an interior resonance $\left(k_{0} a=4.973\right)$ with a much slower speed. In Fig. 3(b), we present the normalized error on the interior surface $S_{\mathrm{int}}, E R R_{S_{\mathrm{int}}}$, as a function of the number of iterations of the conjugate gradient scheme. It appears that the final error on $S_{\mathrm{int}}$ is more indicative for the actual error due to the discretization of integral equation on $S$ and the presence of an interior resonance. At the interior resonance $\left(k_{0} a=4.973\right)$ we clearly observe an increase of the error at iterations where the conjugate gradient scheme adds field components of the interior resonance to the actual surface field.

For $k_{0} a=4.973$, in Fig. 4, we present the absolute value of the surface field $\boldsymbol{\nu} \times \boldsymbol{H}$ as a function of the angular coordi- 


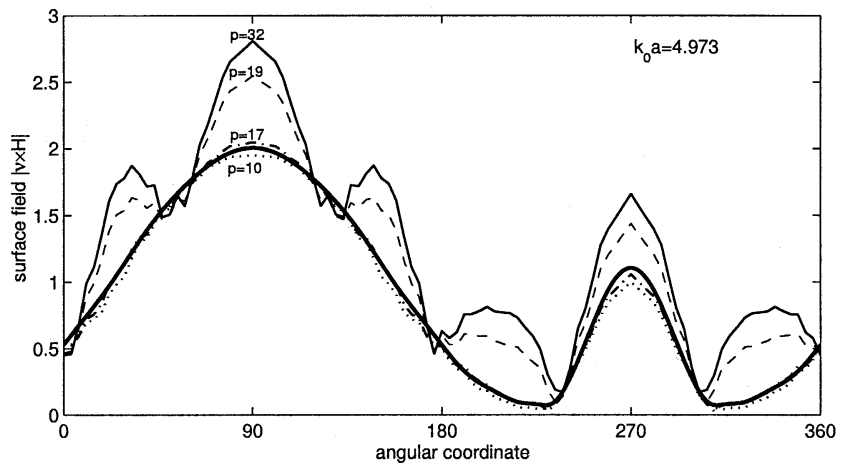

(a)

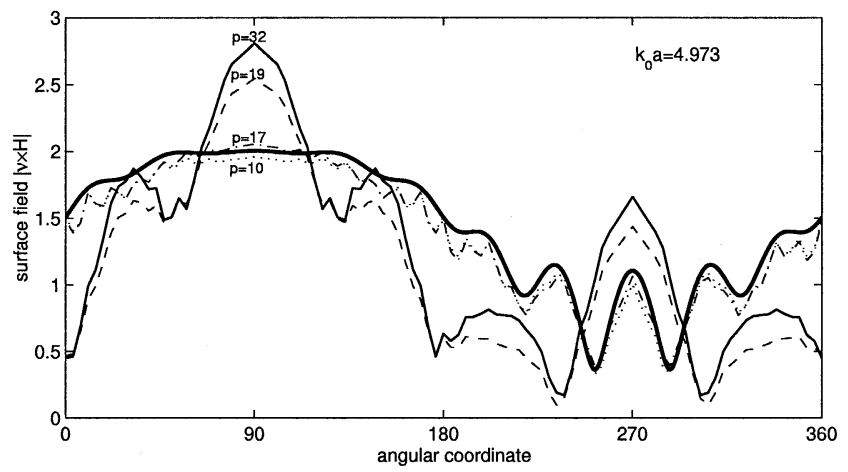

(b)

Fig. 4. (a) Surface field with incident electric field in the $x$ direction and (b) $y$ direction, for increasing number $p$ of iterations; the heavy solid line denotes the Mie series results.

nate along the surface of the sphere in the cross-sectional plane $x=0$, for an increasing number, $p$, of iterations. For comparison, we have also presented the results obtained from an analytical solution of the scattering problem of the sphere in terms of the Mie-series [19]. In accordance with the results of our additional interior error criterion given in Fig. 3(b), we observe, that for increasing number of iterations up to $p=10, \ldots, 17$, the results are approximating the analytical results, but from $p=17$ the surface field starts to deviate. From these results, we draw the conclusion that our additional error criterion seems to be a sufficient error norm to judge the final solution for the surface field $\nu \times H$ on $S$.

\section{Constrained Conjugate Gradient Method}

The present numerical results inspired us to use the interior error norm on $S_{\text {int }}$ as a (sufficient) constraint for the MFIE, that eliminates the occurrence of spurious solutions due to interior resonances. Instead of a conjugate gradient scheme that minimizes the $\mathrm{ERR}_{S}^{2}$, we propose to use a conjugate gradient scheme that minimizes

$$
\mathrm{ERR}^{2}=\mathrm{ERR}_{S}^{2}+\mathrm{ERR}_{S_{\mathrm{int}}}^{2} .
$$

Then, we also need the adjoint operator mapping $S_{\text {int }}$ into $S$, which is obtained (in discrete form) as

$$
\mathcal{L}_{\text {int }}^{*} \boldsymbol{f} \equiv-\boldsymbol{\nu}_{m} \times\left[\boldsymbol{\nu}_{m} \times \sum_{n=1}^{N_{\text {int }}} A_{n} \nabla \overline{\mathcal{G}}\left(\boldsymbol{b}_{n}-\boldsymbol{x}_{\mathrm{m}, \mathrm{j}}\right) \times \boldsymbol{f}\left(\boldsymbol{b}_{n}\right)\right]
$$

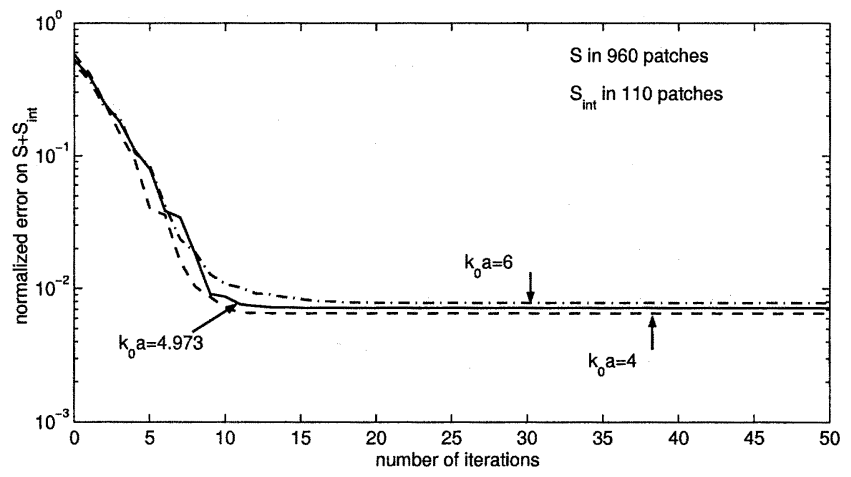

Fig. 5. Normalized error on $S+S_{\text {int }}$ in the constrained conjugate gradient scheme.

In fact, such a constrained conjugate gradient scheme solves iteratively the symmetrized integral equation

$$
\begin{aligned}
\left(\frac{\mathcal{L}^{*} \mathcal{L}}{\left\|\boldsymbol{\nu} \times \boldsymbol{H}^{i}\right\|_{S}^{2}}+\frac{\mathcal{L}_{\text {int }}^{*} \mathcal{L}_{\text {int }}}{\left\|\boldsymbol{H}^{i}\right\|_{S_{\text {int }}}^{2}}\right)[\boldsymbol{\nu} \times \boldsymbol{H}]= & \\
& \frac{\mathcal{L}^{*}\left[\boldsymbol{\nu} \times \boldsymbol{H}^{i}\right]}{\left\|\boldsymbol{\nu} \times \boldsymbol{H}^{i}\right\|_{S}^{2}}+\frac{\mathcal{L}_{\text {int }}^{*} \boldsymbol{H}^{i}}{\left\|\boldsymbol{H}^{i}\right\|_{S_{\text {int }}}^{2}}, \quad \text { on } S .
\end{aligned}
$$

For the nondiscretized problem, this integral equation is uniquely solvable, since the symmetrized operator $\mathcal{L}^{*} \mathcal{L}$ is nonnegative and the symmetrized operator $\mathcal{L}_{\text {int }}^{*} \mathcal{L}_{\text {int }}$ is positive, because the latter operator does not have a null-space. Hence, the complete operator is positive and the conjugate gradient scheme (for the nondiscretized problem) converges to the exact solution [21], even for frequencies coinciding with an eigenfrequncy of interior resonance.

In view of the definition of the two error norms of (11), the extra computation time per iteration to take the second error norm into account is roughly of the order $N_{\text {int }} / 3 N$. It is obvious that we want to choose $N_{\text {int }}$ as small as possible.

\section{A. Numerical Example of a Sphere}

As an example, we again consider the problem of scattering of a plane wave by a sphere. The precise shape of the interior surface is not essential, in fact the only important requirement is that the discretized version of the interior object prescribes a closed interior surface within numerical accuracy. In order to guarantee that in the discretized counterpart of $S_{\text {int }}$ the field variation is computable within the present computer accuracy, we use the same arguments as before and we take care that $S_{\text {int }}$ encloses a sphere with a diameter of a half wavelength, and on $S_{\text {int }}$ we take a discretization of five to six points per wavelength. We start with the same discretization as before and discretize the boundary $S$ of the sphere in 960 patches and the interior surface $S_{\text {int }}$ in 110 patches (see Fig. 2). For the present case, our constrained conjugate gradient method needs only $5 \%$ more computer time than the unconstrained one. We again consider the three frequencies corresponding to $k_{0} a=4,4.973$ and 6 , respectively.

In Fig. 5, we present the combined error, ERR, see (11) as a function of the number of iterations, using the constrained conjugate gradient scheme. We observe that, for $k_{0} a=4.973$, where the unconstrained conjugate gradient methods yields a 
TABLE I

FINAL NORMALIZED ERROR ON $S+S_{\text {int }}$ FOR DIFFERENT DISCRETIZATIONS $\left(k_{0} a=4.973\right)$

\begin{tabular}{l|l|l|l}
\hline \hline & $S$ in 110 patches & $S$ in 960 patches & $S$ in 9800 patches \\
\hline$S_{\text {int }}$ in 110 patches & ERR $=0.0457$ & ERR $=0.0072$ & ERR $=0.0006$ \\
\hline$S_{\text {int }}$ in 960 patches & ERR $=0.0464$ & ERR $=0.0072$ & ERR $=0.0006$ \\
\hline$S_{\text {int in } 9800 \text { patches }}$ & ERR $=0.0465$ & ERR $=0.0072$ & ERR $=0.0006$ \\
\hline \hline
\end{tabular}

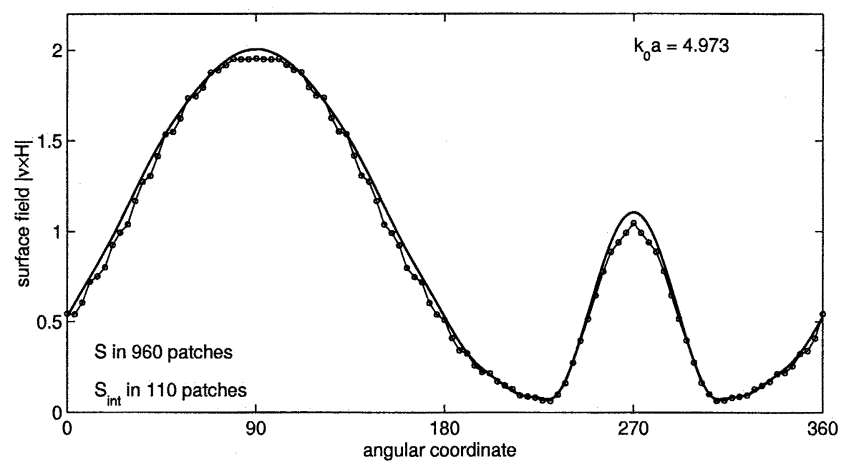

(a)

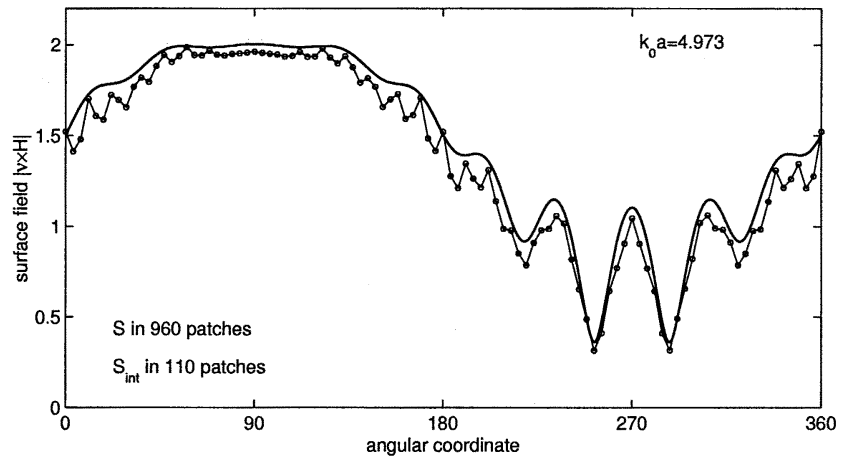

(b)

Fig. 6. (a) Surface field with incident electric field in the $x$ direction and (b) $y$ direction; solid line: Mie series results; circles: results based on minimization of combined error criterion.

spurious solution due to an interior resonance, the error minimized by the constrained conjugate gradient method decreases monotonically to some constant value. It is further observed that the final error (after 50 iterations) increases with increasing $k_{0} a$ and this fact gives the impression that this final error is a measure for the quality of the computed surface field. We therefore investigate the discretization errors in more detail, by taking different discretizations of the boundary surface $S$ of the scattering object and the interior surface $S_{\mathrm{int}}$. In Table I, the combined error ERR is presented for the various discretizations. We observe that the discretization of the interior surface in 110 patches is sufficient. This is roughly a discretization of five to six points per wavelength. In order to be sure to eliminate spurious solutions due to interior resonances, a smaller discretization of this

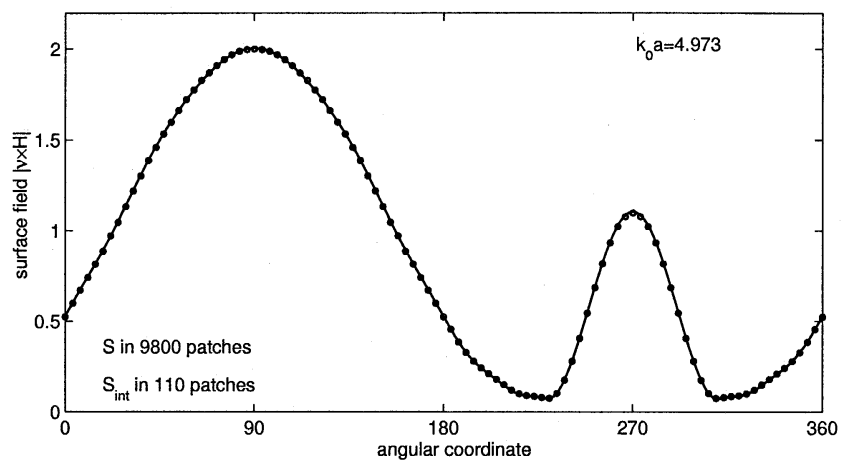

(a)

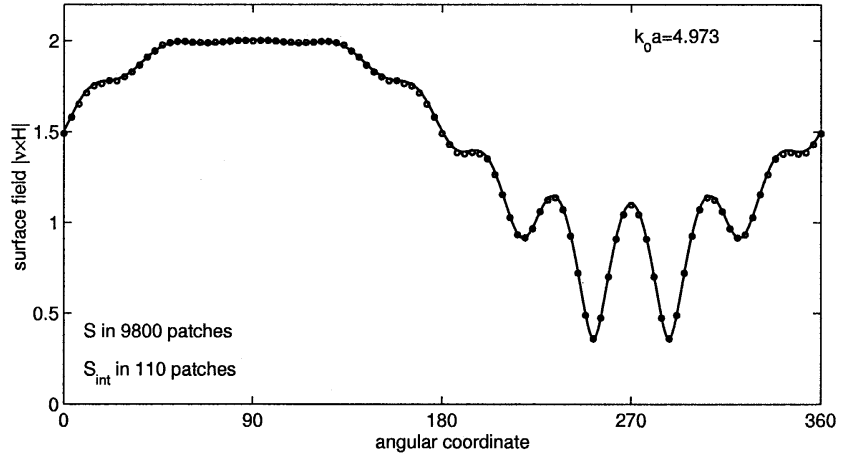

(b)

Fig. 7. (a) Surface field with incident electric field vector in the $x$ direction and (b) $y$ direction; solid line: Mie series results, circles: results based on minimization of combined error criterion.

interior surface is not allowed. Using a discretization of the interior surface in 110 patches, we further observe that the error decreases from 0.0475 to 0.0006 when we refine the discretization of the boundary surface $S$ from 110 patches to 9800 patches, which is roughly a discretization of 2 points per wavelength to 20 points per wavelength, respectively. This confirms our expectation that the normalized error over both $S$ and $S_{\text {int }}$ is a sufficient error criterion, not only theoretically, but also in computational sense.

In Fig. 6, we present the absolute value of the surface field $\boldsymbol{\nu} \times \boldsymbol{H}$. The discrepancies between our numerical results and the Mie series results comply with the final error ERR of about $1 \%$ (see Fig. 5).

Finer Discretization: Subsequently, we study the effect of a finer discretization of the boundary surface $S$. We take a discretization of 9800 patches instead of 960 patches, while keeping the discretization of the interior surface in 110 patches. In Fig. 7, we present the results for the absolute value of the surface field $\boldsymbol{\nu} \times \boldsymbol{H}$. We observe excellent agreement with the analytical solution based on the Mie series. We certainly may conclude that the presence of a spurious field due to interior resonances at the frequency belonging to $k_{0} a=4.973$ has been eliminated by the method of minimizing the combined error on the boundary $S$ and the interior surface $S_{\text {int }}$. In Fig. 8, we present the error as a function of the number of iterations. For comparison we plot the errors for a rough discretization as well. It seems that the combined error is a measure for the error in the discretization of the boundary surface of the object; refining the discretization with a factor of ten yields a reduction 


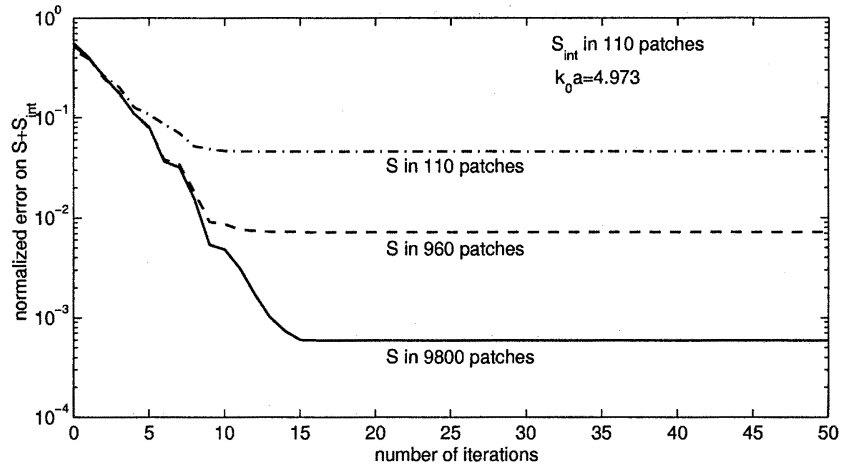

Fig. 8. Normalized error on $S+S_{\text {int }}$ in the constrained conjugate gradient scheme.

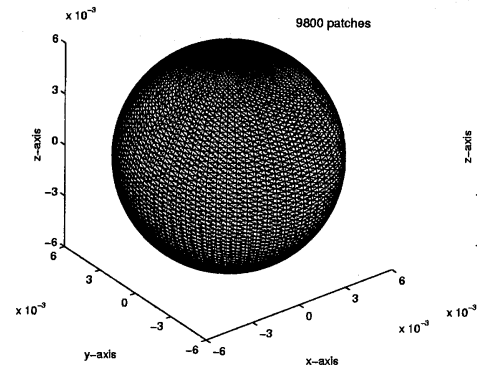

(a)

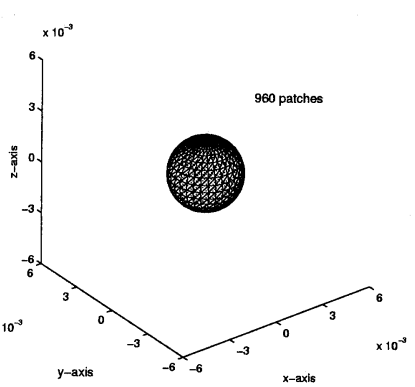

(b)
Fig. 9. (a) Sphere of radius $a$ discretized in 9800 patches and (b) interior sphere of radius $a / 3$ discretized in 960 patches.

of an order in the final error ERR. Further, the error converges very fast (within 20 iterations) to the final error value.

Increased Frequency: In view of the fast convergence, our new, constrained, conjugate gradient scheme offers the possibility to compute the scattered field from objects which are large compared to the wavelength. Therefore, we now take a wave number of $k_{0} a=15$. We take a discretization of 9800 patches on the boundary surface $S$ and 960 points on the interior surface $S_{\text {int }}$, see Fig. 9. This means that on both surfaces we have discretizations of roughly six points per wavelength. In Fig. 10, we present the error as function of the number of iterations for $k_{0} a=15$. For comparison, we present the results for a discretization of the interior surface in 110 patches as well (dotted line). For further comparison we have copied the results for $k a=4.973$ from Fig. 8 and plotted as dashed line. Although, for both cases, the boundary surface of the object is discretized in the same number of points, it seems that the discretization errors, made for higher values of $k a$, becomes relatively smaller. In addition a finer discretization of the interior surface helps to decrease the error after a large number of iterations. This fact is not observed for lower frequencies (see Table I). In Fig. 11, we present, for $k_{0} a=15$, the absolute values of the surface field $\boldsymbol{\nu} \times \boldsymbol{H}$. The discrepancies between our numerical results and the Mie series results comply with the final error ERR of about $0.5 \%$ (see Fig. 10).

\section{NUMERICAL RESUlTS FOR VARIOUS OBJECTS}

So far we have carried out a number of numerical experiments for the sphere to illustrate the performance of the constrained conjugate gradient method. However, one can argue that the

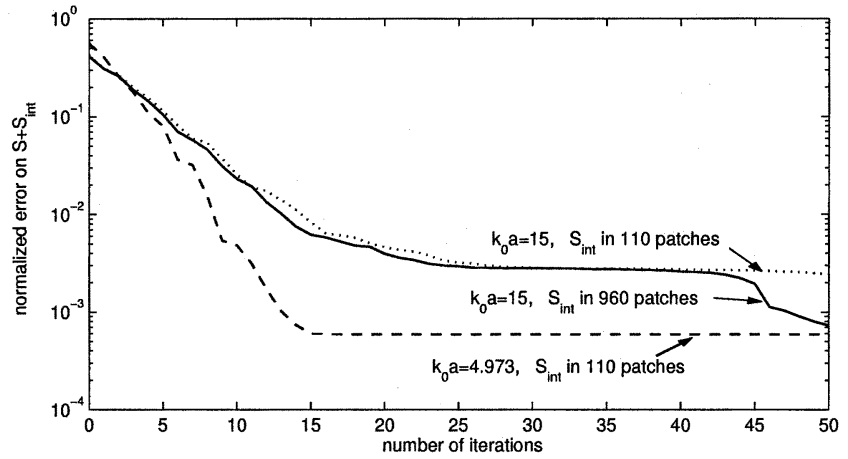

Fig. 10. Normalized error on $S+S_{\text {int }}$ in the constrained conjugate gradient scheme

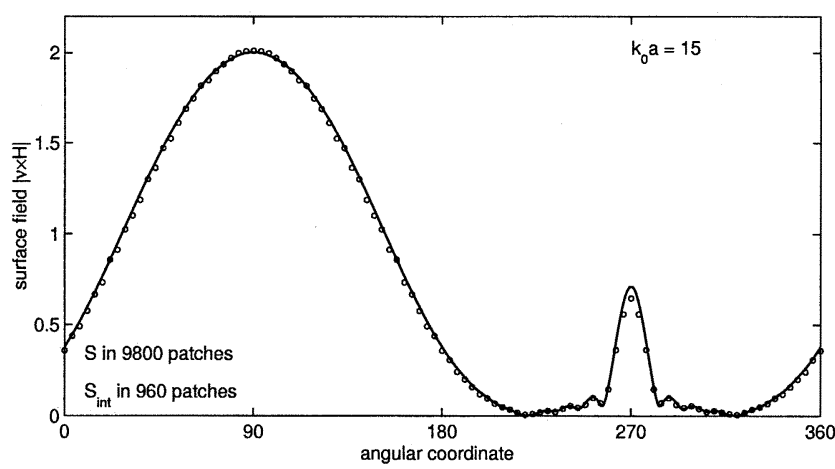

(a)

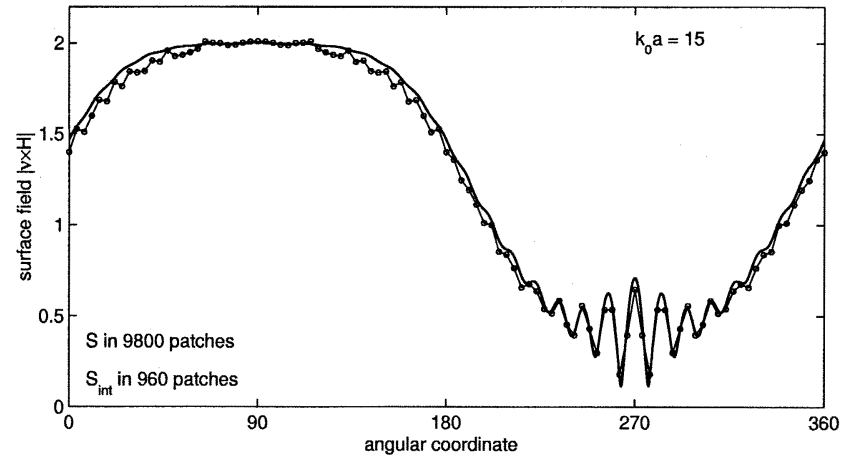

(b)

Fig. 11. Surface field for plane wave incidence with electric field vector in the (a) $x$ direction and (b) $y$ direction; solid line: Mie series results, circles: results based on minimization of combined error criterion.

sphere is a too simple shape to validate the proposition and actual implementation of the combined error norm. We therefore discuss some more experiments with various scattering objects. In all cases, the incident field is a plane wave incident in the negative $z$ direction, while its electric field vector is oriented in the $y$ direction. In view of our practical interest, the frequency of operation is $35.06 \mathrm{GHz}$, so that the wavelength is $0.856 \mathrm{~cm}$. The various objects have discretizations of five to six points a wavelength. The interior surface is always a sphere with a radius of $2 \mathrm{~mm}$, and discretized into 110 patches.

\section{A. Finite Cylinder}

We consider a finite cylinder with circular cross-section. We discretize the boundary surface into 1972 patches [Fig. 12(a)]. The center of the interior sphere coincides with the center of 


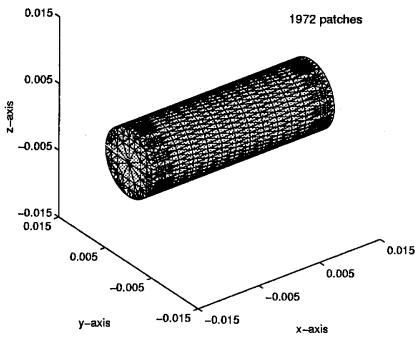

(a)

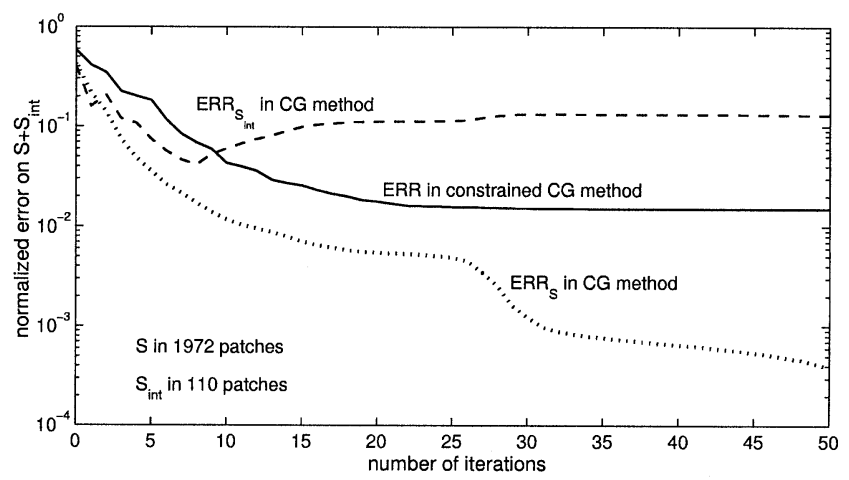

(c)

Fig. 12. (a) $30 \mathrm{~mm}$ cylinder of $5 \mathrm{~mm}$ radius discretized in 1972 patches, (b) interior sphere of $2 \mathrm{~mm}$ radius discretized in 110 patches, and (c) normalized errors in the constrained conjugate gradient scheme.

cylinder [Fig. 12(b)]. We first compute the surface field with the conjugate gradient method dealing with the MFIE only. In Fig. 12(c), the normalized error $\mathrm{ERR}_{S}$ as a function of the number of iterations is presented as the dotted line. The interior error, $\mathrm{ERR}_{S_{\mathrm{int}}}$ is represented by the dashed line. We observe that after 10 iterations the interior error increases, while the boundary error decreases rapidly. From our experience of the previous example we know that this is an indication that we compute a spurious solution due to an interior resonance. When we compute the surface field with our constrained conjugate gradient method that minimizes the combined error, ERR, we observe that the latter scheme converges to a minimum that is a measure for the discretization error [see solid line in Fig. 12(c)].

\section{B. Finite Plate}

Next, we consider a square plate, discretized into 17280 patches (Fig. 13). So far we have located the interior sphere in the center of the targets. In order to show that the location is not important, as long as the interior surface is not too close to the boundary surface of the object (to stay away from the singular point of the Green function), we locate the interior sphere near a corner of the plate (see Fig. 13). We take the center of the interior sphere at $\{-4,-4,0\} \mathrm{cm}$. We first use the unconstrained conjugate gradient method. In Fig. 13, the normalized error, $\mathrm{ERR}_{S}$, as a function of the number of iterations is presented as the dotted line, while the interior error, $\mathrm{ERR}_{S_{\mathrm{int}}}$, is given by the dashed line. We observe that already after five iterations the interior error increases, while the boundary error remains decreasing, so that we again deal with an interior resonance and compute a spurious solution. However, when we use our

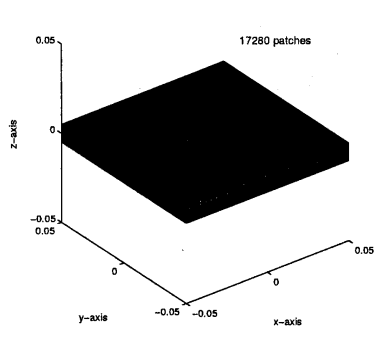

(a)

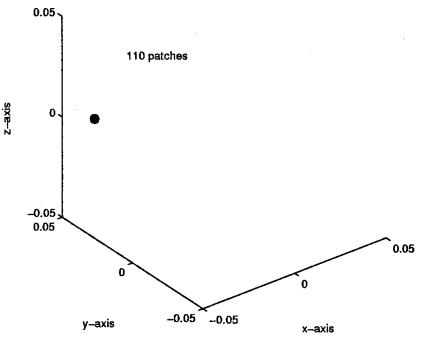

(b)

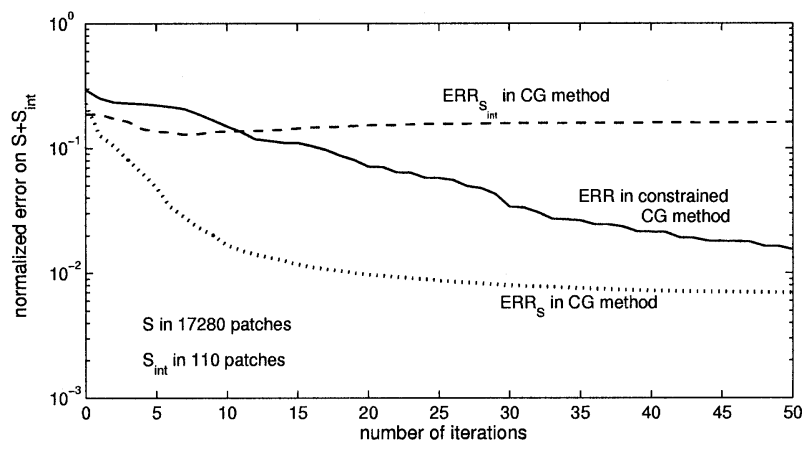

(c)

Fig. 13. (a) Plate of $10 \times 10 \times 1 \mathrm{~cm}^{3}$ discretized in 17280 patches, (b) interior sphere of $2-\mathrm{mm}$ radius discretized in 110 patches, and (c) normalized errors in the constrained conjugate gradient scheme.

constrained conjugate gradient method that minimizes the combined error, ERR, we observe again that the latter scheme converges to a minimum that is a measure for the discretization error [see solid line in Fig. 13(c)]. Although we have many $(2 \times 3 \times 17280)$ unknowns, still the constrained conjugate gradient method reaches its minimum in roughly 50 iterations.

\section{Capped Box}

We finally consider a more complicated object. We take a rectangular box capped with three cones. The box has a length of $2 \mathrm{~cm}$ and a cross-section of $0.8 \times 0.8 \mathrm{~cm}^{2}$. The center of this box coincides with the origin of the coordinate system. At the end, the box is capped with a right circular cone; this cone has a height of $1 \mathrm{~cm}$ and a basis cross-section with a radius of $0.4 \mathrm{~cm}$. In addition on two opposite sides we have placed cones with a height of $0.4 \mathrm{~cm}$ and a basis cross-section with a radius of $0.4 \mathrm{~cm}$. We discretize the boundary surface into 1888 patches [Fig. 14(a)]. To test the method for an extreme case, the interior sphere is chosen to be located outside the rectangular main part of the object. Actually, we locate it inside the cone at the end of the object [Fig. 14(b)]: the center of the interior sphere is at $1.2,0$, and $0 \mathrm{~cm}$. We first use the conjugate gradient method that minimizes the error $\mathrm{ERR}_{\mathrm{S}}$ in the MFIE. In Fig. 14(c), the normalized error $\mathrm{ERR}_{S}$ as a function of the number of iterations is presented as the dotted line. Simultaneously, we check the interior error by presenting the normalized error $\mathrm{ERR}_{S_{\text {int }}}$ as the dashed line. We see that the initial estimate of the physical-optics approximation yields a normalized error close to $100 \%$ and this indicates that the physical-optics approximation without further improvement cannot be used for these types of targets. We further observe that the interior error $\mathrm{ERR}_{S_{\text {int }}}$ after five iterations does not decrease anymore and 


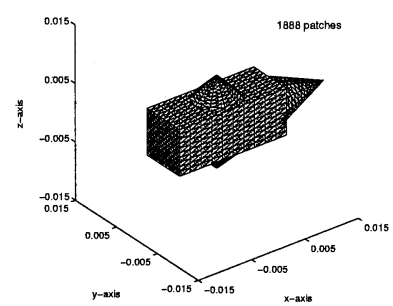

(a)

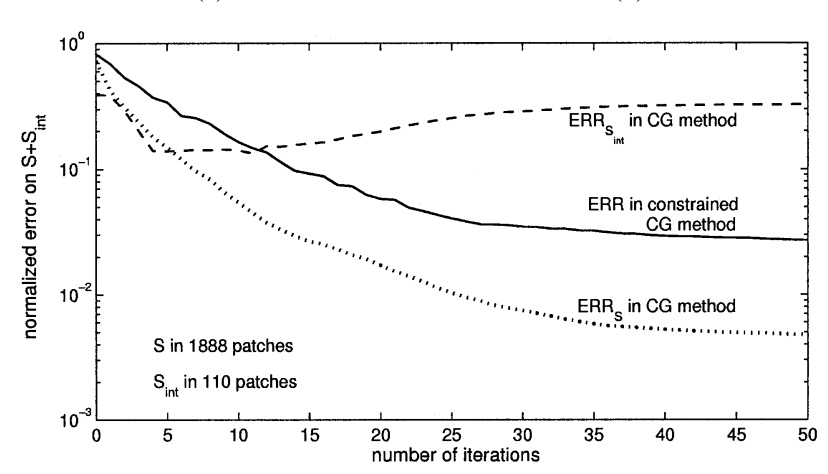

(c)

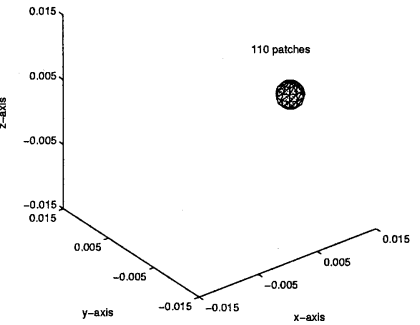

(b)
Fig. 14. (a) Capped box discretized in 1888 patches, (b) interior sphere of $2 \mathrm{~mm}$ radius discretized in 110 patches, and (c) normalized errors in the constrained conjugate gradient scheme.

increases up to an error larger than $30 \%$, while the boundary error $\mathrm{ERR}_{S}$ decreases rapidly. When we use our constrained conjugate gradient method that minimizes the combined error ERR we observe that the latter scheme converges to a minimum that is a measure for the discretization error [see solid line in Fig. 14(c)]. This example shows that for complex objects, where the behavior and occurrence of interior resonances is completely unpredictable, the method dealing with the MFIE only is unreliable, and has to be used in combination with our interior error constraint.

\section{CONCLUSION}

In this paper we have developed a constrained conjugate gradient method for solving the MFIE. In order to restore uniqueness, we use the unique interior (null-field) integral equation over a closed interior surface as a constraint for the conjugate gradient method. To that end the problem is posed as an optimization problem in which a cost functional consisting of two terms are minimized. The first term is the normalized error norm with respect to the boundary integral equation, while the second term is the normalized error with respect to the integral equation over a closed interior surface. In order to limit the extra computation time due to the presence of the constraint, the interior surface has been chosen as small as possible, but such that the field variation over this surface remains visible in computational sense. Numerical experiments indicate that an interior surface enclosing a spherical domain with a radius of about a half wavelength is sufficient. Then, for realistic objects the extra computation time is less than $3 \%$. Numerical examples have shown that the proposed computational method produces stable results even for frequencies corresponding to the interior resonances of the scatterer. Further, we have observed that the constrained conju- gate gradient scheme converges to a minimum that is representative for the discretization error made in the MFIE. Improving the discretization leads to a lower minimum. With this feature we have arrived at a quantitative criterion for the global error made in the discretization. We note that a similar phenomenon occurs in the method using Yaghjian's augmented equations [11].

Although in this paper we handle only the MFIE, we note that the method is also applicable for the case of the EFIE. As constraint one can use the interior equations of either electric type or magnetic type. In view of its simplicity we prefer the latter one.

\section{REFERENCES}

[1] A. J. Poggio and E. K. Miller, "Integral equations for three dimensional scattering problems," in Computer Techniques for Electromagnetics, R. Mittra, Ed. New York: Pergamon, 1973, pp. 159-264.

[2] N. Morita, N. Kumaga, and J. R. Mautz, Integral Equation Methods for Electromagnetics. Boston, MA: Artech House, 1990.

[3] D. Colton and R. Kress, Integral Equations Methods in Scattering Theory. New York: Wiley, 1983.

[4] K. J. Langenberg, "A thorough look at the nonuniqueness of the electromagnetic scattering integral equation solutions as compared to the scalar acoustic ones," Radio Sci., 2002, to be published.

[5] K. K. Mei and J. G. van Bladel, "Scattering by perfectly-conducting rectangular cylinders," IEEE Trans. Antennas Propagat., vol. AP-11, pp. 185-192, Nov. 1963.

[6] M. Hestenes and E. Steifel, "Method of conjugate gradients for solving linear systems," J. Res. Natl. Bureau Standards, vol. 49, pp. 409-436, 1952.

[7] K. M. Mitzner, "Numerical solution of the exterior scattering problem and eigenfrequencies of the interior problem," Dig. URSI Radio Sci. Meeting, p. 75, 1968.

[8] J. R. Mautz and R. F. Harrington, " $H$-field, $E$-field and combinedfield solutions for conducting bodies of revolution," A.E. $\ddot{U}$., vol. 32, pp. 157-163, 1978.

[9] A. F. Peterson, "The interior resonance problem associated with surface integral equations of electromagnetics: Numerical consequences and a survey of remedies," Electromagn., vol. 10, pp. 293-312, 1990.

[10] A. D. Yaghjian, "Augmented electric- and magnetic-field integral equations," Radio Sci., vol. 16, pp. 987-1001, 1981.

[11] S. Markarov and R. Vedantham, "Performance of GMRES iterative solution for the magnetic field integral equation," Radio Sci., to be published.

[12] M. B. Woodworth and A. D. Yaghjian, "Derivation, application and CG solution of dual-surface integral equation equations for three-dimensional, multi-wavelength perfect conductors," in Application of Conjugate Gradient Method to Electromagnetics and Signal Analysis, T. K. Sarkar, Ed. Amsterdam, The Netherlands: Elsevier, 1991, vol. PIER 5, pp. 103-129.

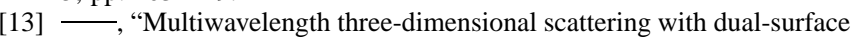
integral equations," J. Opt. Soc. Amer. A, Opt. Image Sci., vol. 11, pp. 1399-1413, 1994

[14] G. W. Oseen, "Über die wechselwirkung zwischen zwei elektrischen dipolen undüber die drehung der polarizationsebene in kristallen und flüssigkeiten," Annalen der Physik, vol. 48, pp. 1-56, 1915.

[15] H. A. Schenck, "Improved integral formulation for acoustic radiation problems," J. Acoust. Soc. Amer., vol. 44, pp. 41-58, 1967.

[16] R. Mittra and C. A. Klein, "Stability and convergence of moment method solutions," in Numerical and Asymptotic Techniques in Electromagnetics, R. Mittra, Ed. New York: Springer-Verlag, 1975, pp. 129-163.

[17] P. C. Waterman, "Numerical solution of electromagnetic scattering problems ," in Computer Techniques for Electromagnetics, R. Mittra, Ed. New York: Pergamon, 1973, pp. 97-157.

[18] P. M. van den Berg, "Iterative computational techniques in scattering based upon the integrated square error criterion," IEEE Trans. Antennas Propagat., vol. AP-32, pp. 1063-1071, Oct. 1984.

[19] P. W. Barber and S. C. Hill, Light Scattering by Particles: Computational Methods, Singapore: World Scientific, 1990.

[20] R. F. Bloemenkamp, A. Abubakar, and P. M. van den Berg, "Inversion of experimental multi-frequency data using the contrast source inversion method," Inverse Problems, vol. 17, pp. 1611-1622, 2001. 
[21] R. E. Kleinman and P. M. van den Berg, "Iterative schemes based on minimization of a uniform error criterion," in Application of Conjugate Gradient Method to Electromagnetics and Signal Analysis, T. K. Sarkar, Ed. Amsterdam, The Netherlands: Elsevier, 1991, vol. PIER 5, pp. 67-102.

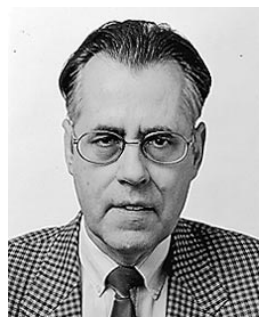

Peter M. van den Berg was born in Rotterdam, The Netherlands, on November 11, 1943. He received the degree in electrical engineering from the Polytechnical School of Rotterdam, Rotterdam, The Netherlands, in 1964, the B.Sc. and M.Sc. degrees in electrical engineering, and the Ph.D. degree in technical sciences from Delft University of Technology, Delft, The Netherlands, in 1966, 1968, and 1971, respectively.

From 1967 to 1968 , he was employed as a Research Engineer by the Dutch Patent Office. Since 1968, he has been a member of the Scientific Staff of the Electromagnetic Research Group, Delft University of Technology. During these years, he carried out research and taught classes in the area of wave propagation and scattering problems. During the academic year 1973 to 1974, he was a Visiting Lecturer in the Department of Mathematics, University of Dundee, Scotland, U.K., financed by an award from the Niels Stensen Stichting, The Netherlands. During a three-month's period from 1980 to 1981, he was a Visiting Scientist at the Institute of Theoretical Physics, Goteborg, Sweden. In 1981, he was appointed as a Professor at the Delft University of Technology. From 1988 to 1994, he carried out research at the Center of Mathematics of Waves, University of Delaware, Newark, these visits were financed by a NATO award. During the summer of 1993 to 1995, he was a Visiting Scientist at Shell Research B.V., Rijswijk, The Netherlands. Since 1994, he has been a Professor at the Delft Research School Center of Technical Geoscience. At present, his main research interest is the efficient computation of field problems using iterative techniques based on error minimization, the computation of fields in strongly inhomogeneous media, and the use of wave phenomena in seismic data processing. Major interest is in an efficient solution of the nonlinear inverse scattering problem.
Erdal Korkmaz was born in Gole, Turkey, on February 10, 1973. He received the M.Sc. degree in electrical engineering from Delft University of Technology, Delft, The Netherlands, in 1997, where he is currently working toward the Ph.D. degree in technical sciences at the Laboratory of Electromagnetic Research.

In 1997, he was a Research Student at Delft Institute of Microelectronics and Submicrontechnology (DIMES). Currently, he is with the TNO Physics and Electronics Laboratory, The Hague, The Netherlands. At present, his main research activity is solving boundary integral equations.

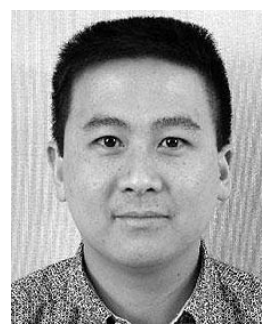

Aria Abubakar (M'97) was born in Bandung, Indonesia, on August 21, 1974. He received the M.Sc. degree (cum laude) in electrical engineering and the $\mathrm{Ph} . \mathrm{D}$. degree (cum laude) in technical sciences, from Delft University of Technology, Delft, The Netherlands, in 1997 and 2000, respectively.

In 1996, he was a Research Student at Shell Research B.V., Rijswijk, The Netherlands. In 1999, he was a Summer Intern at Schlumberger-Doll Research, Ridgefield, CT. From 2000 to 2003, he was a Postdoctoral Applied Researcher at the Laboratory of Electromagnetic Research and Section of Applied Geophysics, Delft University of Technology. Currently, he is a Senior Research Scientist at Schlumberger-Doll Research. His current research activity includes solving forward and inverse problems in acoustics, electromagnetics, and elastodynamics.

Dr. Abubakar is the recipient of the Best Master's Thesis Award in electrical engineering, given by Delft University of Technology in 1997. 